Borah et al. /Journal of Tropical Forestry and Environment Vol. 9, No. 02 (2019) 21-35

\title{
Physiochemical Studies in Seedlings of Teak (Tectona grandis Linn. f.) of North East India in Relation to Drought Resistance for Selection of Improved Germplasm
}

\author{
N. Borah ${ }^{1}$, P.K. Borua ${ }^{2}$, S. Roy ${ }^{3}$ and S.P. Saikia ${ }^{*}$ \\ ${ }^{I}$ Medicinal Aromatic and Economic Plants Group, Biological Sciences and Technology Division, CSIR- \\ North East Institute of Science and Technology, Jorhat, India \\ ${ }^{2}$ Department of Life Sciences, Dibrugarh University, Dibrugarh, India \\ ${ }^{3}$ Division of Genetics and Molecular Biology, CSIR-National Botanical Research Institute Uttar Pradesh, \\ India
}

Date Received: 10-05-2018 Date Accepted: 23-12-2019

\begin{abstract}
The global climate change is occurring at an unpredictable rate, where periods of drought are predicted to be extremely severe. The drought tolerance in Teak (Tectona grandis Linn. f.) accessions; collected from North East India was screened under water stress conditions created by reducing irrigation doses. Parameters targeted for screening were vegetative growth, physiological parameters and chemical constituents of leaves. Water stress treatment revealed that plant height, leaves number/plant, average leaf area, $\mathrm{N}, \mathrm{P}, \mathrm{K}, \mathrm{Ca}, \mathrm{Cl}$ and $\mathrm{Na}$ content were significantly decreased by increasing the level of water stress conditions in all studied accessions. Variations in the physiological parameters among different accessions may be due to different intensities of natural selection acting upon the traits in their natural habitat. The aim of the study was to determine source variation in Tectona grandis Linn. $\mathrm{f}$. accessions collected from 41 locations of North East India and to identify the best sources to be utilised for reforestation and further genetic improvement work. In our study, three promising drought tolerant accessions were screened in a decreasing order of drought tolerance viz. GKU-24, GKU-37 and BNU-10 whereas; the drought stress had the most adverse effect on ASM-124 and LUT-45.
\end{abstract}

Keywords: Drought, Teak, physiochemical responses, forest, India

\section{Introduction}

Water is the most limiting ecological resource for most tree and forest sites. As soil-water content declines, trees become more stressed and begin to react to resource availability changes. A point is reached when water is so inadequately available that tree tissues and processes are damaged. Lack of water eventually leads to catastrophic biological failures and death. Growing periods with little water can lead to decreased rates of diameter and height growth, poor resistance to other stresses, disruption of food production and distribution, and changes to the timing and rate of physiological processes, like fruit production and dormancy. More than eighty percent of the variation in tree growth is because of water supply. The effect is more prominent at early stage of growth. Add to that, the excessive population and livestock pressure and the requirements of forest products for essential development generate a pressure on forest resources like fuel wood, fodder, timber, lumber, paper, etc. which in turn triggers a deforestation process.

*Correspondence: spsaikia@gmail.com

Tel: +919435096977

ISSN 2235-9370 Print/ISSN 2235-9362 Online (C) University of Sri Jayewardenepura 
Overexploitation of the forest's resources as compared to its incremental and regenerative capacities escalates the forest depletion and degradation process. Excessive deforestation has not only local but also global environmental degradation ramifications. It can also affect sustainable socio-economic developmental processes in the developing countries as forests have been generating a lot of employment opportunities in the primary, secondary, and tertiary sectors and have been a source of subsistence to the poorest of the poor in the agricultural economies. Furthermore, inhuman face of deforestation is characterised by the increasing stress on the poorer sections of the society and women, as they have been primarily involved in gathering fuel wood, fodder and water in the traditional village economies. The annual demand for industrial wood is about 28 million cubic meters against the production capacity of 12 million cubic meters. Population pressure is always the underlying cause of overexploitation of the natural resources including forest stock. Possibly, poverty, corruption, weak institutions, and wasteful consumption patterns also combine with the population pressure facilitating depletion and degradation of forest stock having enormous environmental degradation ramifications. Therefore, it is very important to manage the forest in efficient way to meet the ecological need, human need and for better green world. Amongst other various ways and means introduction of new germplasms with improved drought tolerance ability in degraded land will lessen the burden to some extent. Understanding the physiology of drought tolerance at early stage of tree species like teak will be of great help to develop better stock for mass production.

Teak (Tectona grandis Linn. f.) (Lamiaceae) is a tropical tree species. It is one of the world's premier hardwood tree species, highly famous for its quality, durability, dimensional stability, weightlessness and resistance to weathering. It is native to India, Myanmar, Thailand, and Indonesia (Verhaegen et al., 2010; Vaishnaw et al., 2015). Today, there is an urgent need for teak conservation measures, and this is especially important in the light of likely climatic changes in the years to come. Due to population pressure and un favourable biotic factors, teak resources have considerably decreased both in extent as well as in density, quality and quantity over the natural range. Establishing a comparative physiological mechanism that are active in plant system in different genotypes is utmost important to understand the mechanism of drought tolerance of plant species. Teak is a widely adopted species and there are scanty of reports on its physiological studies especially in context to different abiotic stress viz. droughts. The aim of the present work is to screen the drought tolerance and drought sensitive teak plant based on various vegetative and physiological parameters as well as leaves nutrient analysis and to study how the vegetative, physiological and leaf nutrients differ between two contrasting teak plants (drought tolerance and sensitive) as well as the interaction of these factors regarding plant development, to identify the best sources to be utilised for reforestation and future genetic improvement work.

\section{Methodology}

\subsection{Plant growth and water stress treatment}

T. grandis accessions were housed in an experimental greenhouse of CSIR-North East Institute of Science and Technology, Jorhat, Assam, India which falls between $27.35^{\circ}-26.30^{\circ} \mathrm{N}$ latitude and $93.45^{\circ}$ $94.30^{\circ} \mathrm{E}$ longitude and enjoys moderate type of climate. Accessions of $T$. grandis were collected through survey from different regions of North East India, from parent plants chosen randomly from each population, which were about $100 \mathrm{~m}$ apart from each other. Accession from each plant were collected and labeled to maintain their identities. The accessions were maintained in the nursery and 6-month old ownrooted uniformed accession were planted in plastic bags $20 \mathrm{~cm}$ diameter and $30 \mathrm{~cm}$ depth, each bag was filled with a constant weight $(6 \mathrm{~kg})$ of sand soil (in the ratio $1: 1$ by volume) and plants were pruned to single shoot. The sand soil was mainly used because it has low water holding capacity and thus suitable for drought stress studies. The upper portion of the green house was covered with green plastic shade, while the other parts remained open. Two set of bags were arranged in factorial experiment in complete 
randomized design with three replications and each replicate was represented by nine plants. Field capacity was calculated according to Klute (1986) and the irrigation treatments were applied once in an interval of 7 days using normal tap water as follows:

a. Irrigation at $100 \%$ of field capacity (Normal irrigation).

b. Irrigation at $75 \%$ of field capacity.

c. Irrigation at $50 \%$ of field capacity.

d. Irrigation at $25 \%$ of field capacity.

The plants were kept at dehydration stress for a period of 6 months. The drought tolerant and sensitive plants were screened on the basis of repeated measurement of vegetative and physiological characteristics as well as analysing leaves chemical constituents.

\subsection{Vegetative characteristics measurements}

Plant height, leaves number per accession and leaf area were determined to study the effect of water stress on different accessions of $T$. grandis. Plant height was measured $(\mathrm{cm})$ from the soil surface till the top of plant. The number of leaves/accession was recorded. Leaf area was measured with a Leaf Area Meter 211 (Systronics) for five leaves chosen randomly from each plant and expressed as average leaf area per leaf. The leaf area was multiplied by the number of leaves occurring in the plant and was expressed as total leaf area per plant.

\subsection{Physiological characteristics measurements}

Transpiration rate, stomatal conductance, net photosynthetic rate, relative water content, intrinsic water use efficiency, instantaneous water use efficiency, leaf area ratio, specific leaf area, root: shoot ratio and plant biomass were determined to identify the physiological adjustment of T. grandis L.f to water stress treatment. Transpiration rate $\left(\mathrm{mmol} \mathrm{m} \mathrm{m}^{-2} \mathrm{~s}^{-1}\right)$, stomatal conductance $\left(\mathrm{mol} \mathrm{m} \mathrm{m}^{-2} \mathrm{~s}^{-1}\right)$ and net photosynthesis rate $\left(\mu \mathrm{molm}^{-2} \mathrm{~s}^{-1}\right)$ were measured using a Portable Photosynthesis System TPS-2 (PP Systems). Leaf relative water content (RWC) was calculated as (Weatherley, 1950):

$\mathrm{RWC}=[($ fresh weight - dry weight $)+($ turgid weight - dry weight $)]$

Turgid weight was determined by placing samples in distilled water and maintaining them at $5^{\circ} \mathrm{C}$ in darkness until they reached a constant weight. Full turgor was typically reached after 12 hours. Dry weight was obtained after placing the samples in an oven at $70^{\circ} \mathrm{C}$ for 48 hours. Intrinsic water use efficiency $\left(\mu \mathrm{molmol}^{-1}\right)$ was determined as the ratio of net photosynthesis rate to stomatal conductance whereas instantaneous water use efficiency $\left(\mu \mathrm{molmmol}^{-1}\right)$ was determined as the ratio of net photosynthesis rate to transpiration (Petite et al., 2000). Leaf Area Ratio $\left(\mathrm{cm}^{2} \mathrm{~g}^{-1} \mathrm{TDW}\right)$ was calculated (Radford, 1967) by dividing the total leaf area by plant (total) dry weight per plant and expressed in $\mathrm{cm}^{2} \mathrm{~g}^{-}$

${ }^{1}$ whereas specific leaf area $\left(\mathrm{cm}^{2} \mathrm{~g}^{-1} \mathrm{LDW}\right)$ was calculated (Hunt, 1990) by dividing total leaf area by leaf dry weight per plant and the average value was expressed in $\mathrm{cm}^{2} \mathrm{~g}^{-1}$. Root-shoot ratio was calculated as the ratio of the dry weight of root to the dry weight of the shoot. Plant Biomass was determined as the biomass of the shoot and root dried to constant weight in oven at $70 \pm 2^{\circ} \mathrm{C}$.

\subsection{Leaves chemical constituents measurements}

Collected leaves samples were dried after washing several times with tap water, to constant weight at $70^{\circ} \mathrm{C}$ and then was digested in a mixture of sulphuric and perchloric acids according to Piper (1950) and the following determinations was carried out as percent of dry weight. Total nitrogen percentage (\%) was determined using indophenols blue method according to Novozamsky et al. (1974). Phosphorus content was determined by spectrophotometry method according to Temminghoff and Houba (2004). 
Potassium, calcium and sodium were determined by using Flame photometer according to Brown and Lilleland (1946). Chloride was estimated by titration method with silver nitrate according to Jackson (1958). Free proline concentration was measured calorimetrically using ninhydrin reagent according to Bates et al., 1973. Total chlorophyll content in leaves was determined using DMSO (Dimethyl sulphoxide) according to Hiscox and Israelstam (1979).

\subsection{Statistical analysis}

Statistical analysis was done according to the standard procedure (Panse and Shukhatme, 1967). Results were taken to be reported as significant or non-significant based on Least Significant Difference (LSD) test with significance level at $\mathrm{P} \leq 0.05$.

\section{Results and Discussion}

\subsection{Accession source}

Accession source of T. grandis are given in Table 1. A clear cut distinction in the performance of the accessions was observed when grown under limited water conditions (irrigation at $25 \%$ of field capacity); the percentage of survival recorded was 100 in 5 out of the 41 accessions (Table 2), while the rest showed very less survival percentage or failed to survive. Thus these 5 accessions were taken for further study.

\subsection{Vegetative characterization of $T$. grandis in response to drought stress}

Data presented in Table 3 indicated that the plant height was significantly and gradually decreased with decreasing irrigation water at $100 \%$ to $75 \%, 50 \%$ and $25 \%$ from field capacity.

Irrigation at $25 \%$ from field capacity resulted in the lowest values in plant height with ASM-124 followed by LUT-45. Increasing soil moisture stress by decreasing amount of available water of the soil led to a progressive significant reduction in plant height. The effect of stress may be attributed to the loss of turgor which affects the rate of cell expansion and ultimate cell size. Loss of turgor is probably the most sensitive processes to water stress. Consequently, water deficit decreased growth rate, stem elongation, leaf expansion and stomatal aperture. GKU-24 and GKU-37 recorded the highest significant values of plant height (20 and $13 \mathrm{~cm}$ respectively); on the other hand, ASM-124 recorded the lowest values $(5 \mathrm{~cm})$ when irrigated at $25 \%$ from field capacity (Table 3 ). Thus, it can be summarised that plant height was decreased by increasing the level of water stress. Leaves number/accession was significantly affected by water stress conditions in all studied teak accessions. The number of leaves decreased gradually with increasing water depletion up to $25 \%$. In general, in all studied accessions, which received normal $(100 \%)$ or moderate irrigation $(75 \%)$ produced the highest leaves number, while the lowest was produced when plants exposed to drought stresses $(50 \%$ or $25 \%)$. However, under the same conditions of drought stresses, GKU-24 seem to be more tolerant to severe drought stress, resulted in more leaves number in comparison to other studied accessions. The obtained results are in agreement with those of earlier finding where the leaves number was decreased by increasing the level of drought stress (Gowda, 1998; Abd El-Samed, 1995, Hassan, 1998 and Shaheen et al., 2011). The average leaf area was significantly decreased with increasing drought stresses (Table 3). The accessions which were irrigated with normal irrigation (100\%) produced the highest leaf area, while the lowest was recorded when accessions were irrigated with $25 \%$ of the field capacity. However, LUT-45 produced the lowest leaf area when exposed to severe drought stress $(25 \%)$, while the highest leaf area was obtained with BNU-10 irrigated with normal irrigation (100\%). Drought stress reduced plant growth by affecting various physiological and biochemical processes (Jaleel et al., 2008a,b; Farooq et al., 2008); also, it affects both elongation and expansion growth (Kusaka et al., 2005; Shao et al. 2008). 
Table 1: Source detail of $T$. grandis and their geographical locations.

\begin{tabular}{|c|c|c|c|c|c|c|c|c|}
\hline \multirow{2}{*}{$\begin{array}{l}\text { Accession } \\
\text { Code }\end{array}$} & \multirow{2}{*}{ State } & \multirow{2}{*}{ Locality } & \multirow{2}{*}{$\begin{array}{l}\text { Latitude } \\
(\mathrm{N})\end{array}$} & \multirow{2}{*}{$\begin{array}{l}\text { Longitud } \\
\text { e (E) }\end{array}$} & \multirow{2}{*}{$\begin{array}{l}\text { Altitude } \\
(\mathrm{m})\end{array}$} & \multirow{2}{*}{$\begin{array}{l}\text { Rain } \\
(\mathrm{mm})\end{array}$} & \multicolumn{2}{|c|}{ Temp $\left({ }^{\circ} \mathrm{C}\right)$} \\
\hline & & & & & & & $\min$ & $\max$ \\
\hline$B N U-10$ & Tripura & Baramura & 23.83 & 91.65 & 904.00 & 2,800 & 8.5 & 33.0 \\
\hline$B N U-11$ & Tripura & Udaipur & 23.31 & 91.31 & 24.68 & 2,100 & 12.0 & 35.0 \\
\hline$B N U-14$ & Tripura & Agartala & 23.50 & 91.25 & 12.80 & 2,240 & 10.0 & 35.0 \\
\hline$B N U-15$ & Tripura & Kamalasagar & 23.63 & 91.25 & $1,000.00$ & 2,700 & 8.1 & 34.3 \\
\hline$G K U-20$ & Nagaland & Tuli & 26.44 & 94.65 & $1,325.00$ & 2,330 & 9.3 & 28.0 \\
\hline$G K U-14$ & Nagaland & Kohima & 25.40 & 94.08 & $1,433.00$ & 2,300 & 4.0 & 31.0 \\
\hline$G K U-16$ & Nagaland & Namsa & 26.78 & 94.77 & 897.64 & 1,644 & 5.3 & 25.5 \\
\hline$G K U-24$ & Nagaland & Mokokchang & 26.50 & 94.75 & $1,322.00$ & 2,328 & 9.0 & 28.0 \\
\hline$G K U-37$ & Nagaland & Wokha & 26.09 & 94.25 & $1,313.69$ & 2,293 & 9.0 & 25.0 \\
\hline СHM-30 & Mizoram & Kolasib & 24.13 & 92.40 & 660.54 & 2,860 & 7.0 & 32.0 \\
\hline CHM-34 & Mizoram & Aizawl & 23.36 & 93.00 & $1,132.00$ & 3,000 & 11.0 & 30.0 \\
\hline CHM-37 & Mizoram & Luntui & 23.66 & 92.92 & 720.00 & 2,790 & 7.1 & 32.3 \\
\hline LUT-39 & Meghalaya & Watrigithim & 25.98 & 90.68 & $1,496.00$ & 3,350 & 5.0 & 37.0 \\
\hline LUT-4O & Meghalaya & Garo Hills & 25.30 & 90.13 & 870.00 & 2,600 & 7.0 & 30.0 \\
\hline LUT-45 & Meghalaya & West Khasi Hills & 25.98 & 90.68 & $1,496.00$ & 3,350 & 5.0 & 37.5 \\
\hline$I A G-16$ & Manipur & Senapati & 24.21 & 93.29 & $1,061.00$ & 1,850 & 3.3 & 34.0 \\
\hline$I A G-52$ & Manipur & Imphal & 24.44 & 93.65 & 790.00 & 990 & 5.0 & 35.0 \\
\hline$I A G-37$ & Manipur & Loktak & 24.30 & 93.55 & 768.0 & 1,183 & 6.0 & 32.0 \\
\hline$I A G-64$ & Manipur & Lamphelpet & 24.44 & 93.65 & 790.00 & 2,027 & 3.2 & 34.5 \\
\hline PUK-18 & Arunachal Pradesh & Domukh & 27.09 & 93.43 & 210.00 & 2,680 & 8.0 & 35.0 \\
\hline PUK-10 & Arunachal Pradesh & Itanagar & 27.06 & 93.41 & 146.00 & 3,000 & 8.0 & 32.0 \\
\hline PUK-87 & Arunachal Pradesh & Naharlagun & 27.00 & 93.42 & 200.00 & 2,688 & 8.0 & 32.0 \\
\hline PUK-67 & Arunachal Pradesh & Roing & 28.05 & 95.89 & 300.00 & 2,800 & 5.0 & 29.0 \\
\hline PUK-91 & Arunachal Pradesh & Balijan & 27.03 & 93.40 & 210.00 & 2,683 & 8.0 & 35.1 \\
\hline ASM-34 & Assam & Golaghat & 26.75 & 94.25 & 188.00 & 1,952 & 9.3 & 39.8 \\
\hline ASM-105 & Assam & Dergaon & 26.73 & 94.01 & 188.00 & 1,952 & 9.3 & 39.8 \\
\hline$A S M-124$ & Assam & Karbi Anglong & 26.04 & 93.67 & 186.00 & 1,974 & 8.02 & 37.0 \\
\hline ASM-16 & Assam & Sengeliati & 26.45 & 97.30 & 126.70 & 1,872 & 8.8 & 38.9 \\
\hline ASM-54 & Assam & Khakarpur & 26.55 & 90.58 & 173.31 & 1,614 & 8.9 & 39.7 \\
\hline ASM-29 & Assam & Titabor & 26.64 & 94.20 & 192.00 & 1,993 & 9.0 & 38.2 \\
\hline ASM-92 & Assam & Marigaon & 26.15 & 92.20 & 189.00 & 1,973 & 10.0 & 38.3 \\
\hline ASM-11 & Assam & Bongaigaon & 26.15 & 90.34 & 53.00 & 3,500 & 12.9 & 31.7 \\
\hline ASM-26 & Assam & Nagaon & 26.45 & 92.41 & 69.00 & 1,745 & 10.0 & 35.0 \\
\hline ASM-89 & Assam & Sonitpur & 26.60 & 92.78 & 86.00 & 1,563 & 11.0 & 31.0 \\
\hline$A S M-6$ & Assam & Dibrugarh & 27.28 & 94.55 & 108.00 & 2,758 & 10.0 & 31.0 \\
\hline ASM-69 & Assam & Dhubri & 26.02 & 89.58 & 34.00 & 1,600 & 8.0 & 30.0 \\
\hline ASM-84 & Assam & Lakhimpur & 27.65 & 96.25 & 87.00 & 2,635 & 8.0 & 31.5 \\
\hline$A S M-46$ & Assam & Sivasagar & 27.00 & 94.36 & 97.00 & 2,504 & 7.0 & 29.0 \\
\hline ASM-62 & Assam & Tezpur & 26.37 & 92.47 & 79.00 & 1,600 & 7.0 & 36.0 \\
\hline$A S M-75$ & Assam & Hajo & 25.31 & 23.11 & 55.00 & 1,800 & 10.0 & 38.0 \\
\hline ASM-51 & Assam & Jorhat & 26.30 & 94.30 & 116.00 & 2,244 & 9.0 & 39.0 \\
\hline
\end{tabular}


Table 2: Survival rate of T. grandis

\begin{tabular}{|c|c|c|c|c|c|}
\hline $\begin{array}{l}\text { Accession } \\
\text { Code }\end{array}$ & $\begin{array}{c}\text { Survival } \\
(\%)\end{array}$ & $\begin{array}{l}\text { Accession } \\
\text { Code }\end{array}$ & $\begin{array}{c}\text { Survival } \\
(\%)\end{array}$ & $\begin{array}{l}\text { Accession } \\
\text { Code }\end{array}$ & $\begin{array}{c}\text { Survival } \\
(\%)\end{array}$ \\
\hline$B N U-10$ & 100.00 & LUT-45 & 100.00 & $A S M-75$ & 5.55 \\
\hline$B N U-11$ & 0.00 & $A S M-124$ & 100.00 & ASM-51 & 0.00 \\
\hline$B N U-14$ & 22.22 & ASM-16 & 0.00 & $I A G-16$ & 0.00 \\
\hline$B N U-15$ & 0.00 & ASM-54 & 0.00 & $I A G-52$ & 0.00 \\
\hline$G K U-20$ & 10.00 & ASM-29 & 0.00 & $I A G-37$ & 14.44 \\
\hline$G K U-14$ & 0.00 & ASM-92 & 10.88 & $I A G-64$ & 0.00 \\
\hline$G K U-16$ & 0.00 & ASM-11 & 0.00 & PUK-18 & 0.00 \\
\hline$G K U-24$ & 100.00 & ASM-26 & 0.00 & PUK-10 & 16.25 \\
\hline$G K U-37$ & 100.00 & ASM-89 & 0.00 & $P U K-87$ & 0.00 \\
\hline CHM-30 & 10.45 & $A S M-6$ & 0.00 & PUK-67 & 0.00 \\
\hline СHM-34 & 0.00 & ASM-69 & 16.44 & PUK-91 & 0.00 \\
\hline CHM-37 & 12.25 & ASM-84 & 0.00 & $A S M-34$ & 0.00 \\
\hline LUT-39 & 0.00 & ASM-46 & 0.00 & ASM-105 & 0.00 \\
\hline LUT-40 & 0.00 & ASM-62 & 0.00 & & \\
\hline
\end{tabular}

\subsection{Physiological characterisation of T. grandis L.f. in response to drought stress}

Transpiration is one of the major gas exchange related traits associated with plant growth and productivity. In tree species stomatal transpiration contributes more than $90 \%$ of total transpiration (Taiz and Zeiger, 2002). Teak have been shown to precisely regulate transpiration rate via stomatal movements (Bolhar-Nordenkanpf, 1987) allowing this species to take advantage of favorable conditions through enhanced $\mathrm{CO}_{2}$ uptake (Fordyce et al., 1995), especially when exposed to significant seasonal fluctuations (Greenwood et al., 2003). The results in Table 4 showed that transpiration rate (E) was negatively affected by water stress and their decline under water stress was significantly higher in ASM-124 (1.51 mmolm $\mathrm{mm}^{-2}$ $\left.{ }^{1}\right)$ followed by LUT-45 and BNU-10 (1.63 and $1.68 \mathrm{mmolm}^{-2} \mathrm{~s}^{-1}$ respectively). Like transpiration rate (E), stomatal conductance (gs) was also negatively affected by water stress, which decreased significantly with increasing water stress (Table 4). GKU-24 and GKU-37 recorded the highest stomatal conductance $(0.19$ molm $\mathrm{s}^{-1}$ ) when exposed to severe drought stress (25\%), while ASM-124 recorded the lowest stomatal conductance $\left(0.07 \mathrm{molm}^{-2} \mathrm{~s}^{-1}\right)$ under similar condition. The results in Table 4 also showed that like $\mathrm{E}$ and gs the Net Photosynthetic rate $(\mathrm{Pn})$ also decreased significantly with increasing drought treatments. The highest Pn was observed in GKU-24 $\left(5.13 \mu\right.$ molm $\left.^{-2} \mathrm{~s}^{-1}\right)$ followed by GKU-37 $\left(2.0 \mu \mathrm{molm}^{-2} \mathrm{~s}^{-1}\right)$ when exposed to severe drought $(25 \%)$ whereas the lowest Pn was observed in ASM-124 $\left(1.09 \mu \mathrm{molm}^{-2} \mathrm{~s}^{-1}\right)$ followed by BNU-10 $\left(1.11 \mu\right.$ molm $\left.^{-2} \mathrm{~s}^{-1}\right)$. Reductions in the photosynthetic activity induced by drought were first triggered by stomatal closure, resulting in limitation of ambient $\mathrm{CO}_{2}$ diffusion to the mesophyll and thus reduction of photosynthesis. Leaf Relative Water Content (RWC) decreased significantly with increasing drought (Table 5). Irrigation at $25 \%$ from field capacity resulted in the lowest values in RWC with ASM-124 (39.68\%) followed by LUT-45. GKU-37 and BNU-10 recorded the highest significant value of RWC (43.88 and 40.88\%). Drought stress-induced decrease in RWC has been reported in many previous studies (Duan et al., 2005; Elsheery and Coa, 2008). Data presented in Table 5 indicated that the Intrinsic Water Use Efficiency and Instantaneous Water Use Efficiency decreased gradually with increasing water depletion up to $25 \%$. However, at $25 \%$ from field capacity GKU-24 recorded the highest Intrinsic Water Use Efficiency $\left(160.77 \mu \mathrm{molmol}^{-1}\right)$ and also showed highest Instantaneous Water Use Efficiency $\left(3.17 \mu \mathrm{molmmol}^{-1}\right)$ followed by GKU-37 and in both, the lowest was recorded in ASM-124. The higher is the value; the better is the efficiency of the plant to divert water for photosynthesis than transpiration. Transpiration and photosynthesis are two major gas exchange parameters, which determine WUE of plants. 
Borah et al. /Journal of Tropical Forestry and Environment Vol. 9, No. 02 (2019) 21-35

Table 3: Effect of water stress treatments on plant height, leaves numbers/accessions and leaves area.

\begin{tabular}{|c|c|c|c|c|c|c|c|c|c|c|c|c|}
\hline \multirow{3}{*}{$\begin{array}{c}\text { Accession } \\
\text { Code }\end{array}$} & \multicolumn{4}{|c|}{ Plant height $(\mathrm{cm})$} & \multicolumn{4}{|c|}{ Leaves numbers/accessions } & \multicolumn{4}{|c|}{ Leaf area $\left(\mathrm{cm}^{2}\right)$} \\
\hline & \multicolumn{4}{|c|}{ Irrigation treatments } & \multicolumn{4}{|c|}{ Irrigation treatments } & \multicolumn{4}{|c|}{ Irrigation treatments } \\
\hline & $100 \%$ & $75 \%$ & $50 \%$ & $25 \%$ & $100 \%$ & $75 \%$ & $50 \%$ & $25 \%$ & $100 \%$ & $75 \%$ & $50 \%$ & $25 \%$ \\
\hline GKU-37 & 20 & 18 & 13 & 13 & 15 & 12 & 10 & 9 & 4.7 & 3.9 & 3.3 & 3.0 \\
\hline LUT-45 & 13 & 13 & 10 & 9 & 13 & 9 & 9 & 8 & 4.3 & 3.1 & 3.1 & 2.5 \\
\hline BNU-10 & 15 & 13 & 12 & 12 & 10 & 11 & 8 & 7 & 5.0 & 3.7 & 3.1 & 2.6 \\
\hline Mean & 16 & 15 & 13 & 12 & 13 & 11 & 9 & 8 & 4.5 & 3.6 & 3.3 & 2.8 \\
\hline SD & 4.98 & 5.75 & 5.04 & 4.96 & 2.87 & 2.23 & 2.32 & 2.93 & 0.53 & 0.46 & 0.30 & 0.21 \\
\hline $\mathrm{CV}$ & 0.31 & 0.38 & 0.39 & 0.41 & 0.22 & 0.20 & 0.26 & 0.36 & 0.12 & 0.12 & 0.10 & 0.07 \\
\hline \multirow[t]{2}{*}{ SEM } & 2.22 & 2.56 & 2.25 & 2.21 & 1.28 & 0.99 & 1.03 & 1.31 & 0.24 & 0.20 & 0.13 & 0.10 \\
\hline & \multicolumn{4}{|c|}{ LSD value at $0.05=1.88$} & \multicolumn{4}{|c|}{ LSD value at $0.05=1.49$} & \multicolumn{4}{|c|}{ LSD value at $0.05=0.55(\mathrm{NS})$} \\
\hline
\end{tabular}

$\mathrm{SD}=$ Standard deviation; $\mathrm{CV}=$ coefficient of variance; $\mathrm{SEM}=$ Standard error of mean.

Table 4: Effect of water stress treatments on stomatal conductance $\left(\mathrm{mol} \mathrm{m}^{-2} \mathrm{~s}^{-1}\right)$, net photosynthetic rate $\left(\mu \mathrm{mol} \mathrm{m} \mathrm{s}^{-2}\right)$ and transpiration rate $\left(\mathrm{mmol} \mathrm{m}^{-2} \mathrm{~s}^{-1}\right)$.

\begin{tabular}{|c|c|c|c|c|c|c|c|c|c|c|c|c|}
\hline \multirow{3}{*}{$\begin{array}{l}\text { Accession } \\
\text { Code }\end{array}$} & \multicolumn{4}{|c|}{$\begin{array}{c}\text { Stomatal conductance } \\
\left(\mathrm{mol} \mathrm{m}^{-2} \mathrm{~s}^{-1}\right)\end{array}$} & \multicolumn{4}{|c|}{$\begin{array}{l}\text { Net photosynthetic rate } \\
\left(\mu \mathrm{mol} \mathrm{m} \mathrm{m}^{-2} \mathrm{~s}^{-1}\right)\end{array}$} & \multicolumn{4}{|c|}{$\begin{array}{l}\text { Transpiration rate } \\
\left(\mathrm{mmol} \mathrm{m}^{-2} \mathrm{~s}^{-1}\right)\end{array}$} \\
\hline & \multicolumn{4}{|c|}{ Irrigation treatments } & \multicolumn{4}{|c|}{ Irrigation treatments } & \multicolumn{4}{|c|}{ Irrigation treatments } \\
\hline & $100 \%$ & $75 \%$ & $50 \%$ & $25 \%$ & $100 \%$ & $75 \%$ & $50 \%$ & $25 \%$ & $100 \%$ & $75 \%$ & $50 \%$ & $25 \%$ \\
\hline GKU-37 & 0.28 & 0.24 & 0.19 & 0.19 & 8.0 & 5.64 & 3.32 & 2.0 & 2.03 & 1.88 & 1.83 & 1.78 \\
\hline GKU-24 & 0.46 & 0.25 & 0.19 & 0.19 & 11.23 & 8.62 & 6.34 & 5.13 & 1.89 & 1.86 & 1.86 & 1.93 \\
\hline LUT-45 & 0.09 & 0.16 & 0.19 & 0.14 & 6.35 & 2.32 & 2.13 & 1.12 & 1.86 & 1.83 & 1.73 & 1.63 \\
\hline BNU-10 & 0.14 & 0.17 & 0.16 & 0.14 & 3.19 & 4.06 & 2.09 & 1.11 & 1.77 & 1.75 & 1.73 & 1.68 \\
\hline ASM-124 & 0.07 & 0.11 & 0.07 & 0.07 & 4.16 & 2.06 & 1.02 & 1.09 & 1.80 & 1.53 & 1.51 & 1.51 \\
\hline Mean & 0.21 & 0.19 & 0.16 & 0.15 & 6.59 & 4.54 & 2.98 & 2.09 & 1.87 & 1.77 & 1.73 & 1.71 \\
\hline SD & 0.15 & 0.04 & 0.04 & 0.04 & 2.86 & 2.41 & 1.83 & 1.56 & 0.09 & 0.13 & 0.12 & 0.14 \\
\hline $\mathrm{CV}$ & 0.71 & 0.21 & 0.25 & 0.27 & 0.43 & 0.53 & 0.61 & 0.75 & 0.05 & 0.07 & 0.07 & 0.08 \\
\hline \multirow[t]{2}{*}{ SEM } & 0.07 & 0.02 & 0.02 & 0.02 & 1.27 & 1.07 & 0.82 & 0.70 & 0.04 & 0.06 & 0.05 & 0.06 \\
\hline & \multicolumn{4}{|c|}{ LSD value at $0.05=0.09$} & \multicolumn{4}{|c|}{ LSD value at $0.05=1.35$} & \multicolumn{4}{|c|}{ LSD value at $0.05=0.09$} \\
\hline
\end{tabular}

$\mathrm{SD}=$ Standard deviation; $\mathrm{CV}=$ coefficient of variance; $\mathrm{SEM}=$ Standard error of mean.

Table 5: Effect of water stress treatments on relative water content $(\%)$, intrinsic water use efficiency $\left(\mu \mathrm{mol} \mathrm{mol}^{-1}\right)$ and instantaneous water use efficiency $\left(\mu \mathrm{mol} \mathrm{mmol}{ }^{-1}\right)$.

\begin{tabular}{|c|c|c|c|c|c|c|c|c|c|c|c|c|}
\hline \multirow[t]{2}{*}{$\begin{array}{l}\text { Accession } \\
\text { Code }\end{array}$} & \multicolumn{4}{|c|}{$\begin{array}{c}\text { Relative water content } \\
(\%)\end{array}$} & \multicolumn{4}{|c|}{$\begin{array}{c}\text { Intrinsic water use efficiency } \\
\left(\mu \mathrm{mol} \mathrm{mol}{ }^{-1}\right) \\
\text { Irrigation treatments }\end{array}$} & \multicolumn{4}{|c|}{ 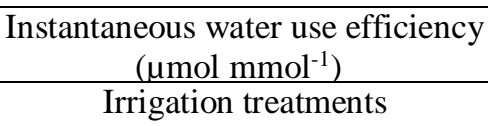 } \\
\hline & $100 \%$ & $75 \%$ & $50 \%$ & $25 \%$ & $100 \%$ & $75 \%$ & $50 \%$ & $25 \%$ & $100 \%$ & $75 \%$ & $50 \%$ & $25 \%$ \\
\hline GKU-37 & 70.34 & 67.75 & 51.17 & 43.88 & 358.10 & 116.54 & 115.94 & 114.92 & 8.17 & 5.26 & 3.76 & 2.90 \\
\hline GKU-24 & 69.14 & 60.53 & 48.65 & 40.10 & 363.48 & 199.00 & 163.41 & 160.77 & 10.46 & 6.91 & 3.85 & 2.96 \\
\hline UT-45 & 67.74 & 59.33 & 47.25 & 39.68 & 287.83 & 97.80 & 89.30 & 65.88 & 7.57 & 4.58 & 3.66 & 2.88 \\
\hline BNU-10 & 66.54 & 57.93 & 46.05 & 40.88 & 281.48 & 52.96 & 52.09 & 51.58 & 7.44 & 4.21 & 3.65 & 2.01 \\
\hline ASM-124 & 64.94 & 56.53 & 44.65 & 39.68 & 276.25 & 40.23 & 38.22 & 34.89 & 7.21 & 3.88 & 3.18 & 1.96 \\
\hline Mean & 61.74 & 60.41 & 47.55 & 40.84 & 313.42 & 101.31 & 91.79 & 85.61 & 8.17 & 4.97 & 3.62 & 2.58 \\
\hline SD & 1.90 & 3.90 & 2.24 & 1.58 & 38.88 & 56.31 & 45.09 & 46.10 & 1.19 & 1.07 & 0.23 & 0.05 \\
\hline $\mathrm{CV}$ & 0.03 & 0.06 & 0.05 & 0.04 & 0.12 & 0.55 & 0.50 & 0.54 & 0.15 & 0.21 & 0.06 & 0.19 \\
\hline \multirow[t]{2}{*}{ SEM } & 0.85 & 1.74 & 1.00 & 0.71 & 17.36 & 25.14 & 20.13 & 20.58 & 0.53 & 0.48 & 0.10 & 0.22 \\
\hline & \multicolumn{4}{|c|}{ LSD value at $0.05=1.97$} & \multicolumn{4}{|c|}{ LSD value at $0.05=19.59$} & \multicolumn{4}{|c|}{ LSD value at $0.05=0.82$} \\
\hline
\end{tabular}

$\mathrm{SD}=$ Standard deviation; $\mathrm{CV}=$ coefficient of variance; $\mathrm{SEM}=$ Standard error of mean 
The water deficit did not significantly affect the leaf area ratio (LAR), however, specific leaf area (SLA) increased significantly in plants submitted to decreasing irrigation water at $25 \%$ in comparis on to $100 \%$ and those under $75 \%$ and $50 \%$ (Table 6). GKU-24 recorded the highest LAR and SLA when subjected to irrigated treatment under all conditions. The root: shoot ratio did not differ significantly between treatments. GKU-24 recorded the highest root: shoot ratio when subjected to irrigated treatment under all conditions (Table 6).

Data presented in Table 7 indicated that biomass partitioning to leaves, shoot and root decreased gradually with increasing water depletion up to $25 \%$. GKU-24 recorded the highest biomass partitioning to leaves, root and shoot when subjected to decreasing irrigated water at $25 \%(16.4 \%, 35.0 \%$ and $49.2 \%$ respectively).

\subsection{Chemical characterisation of T. grandis L.f. in response to drought stress}

Table 8 showed that an appreciable significant reduction in leaf N\% due to increasing drought stress. The lowest nitrogen percentage was obtained at irrigation with $25 \%$ from field capacity in ASM$124(1.78 \%)$, while the highest was obtained with GKU-24 (2.20\%). These results are in harmony with that reported by Moustafa (2002). The nitrogen status of a plant has a significant influence over its water relation, as nitrogen and water often interact. When the soil faces a prolonged period of drought, nitrogen mobility is severely restricted by the dehydrated soil. Thus, when a plant faces water deficit, nitrogen deficiency occurs (DaMatta et al., 2002), which rapidly inhibits plant growth. According to Bänziger et al. (2000), about $50 \%$ of all $\mathrm{N}$ in the leaf is directly involved in photosynthesis as either enzymes or chlorophyll. Thus, if the $\mathrm{N}$ supply is insufficient, photosynthesis is decreased by reducing the leaf area and photosynthesis rate as well as accelerating leaf senescence. The phosphorus content in leaves gradually decreased by decreasing water depletion from 100 to $25 \%$ from field capacity (Table 8 ). Table 6: Effect of water stress treatments on leaf area ratio $\left(\mathrm{cm}^{2} \mathrm{~g}^{-1} \mathrm{TDW}\right)$, specific leaf area $\left(\mathrm{cm}^{2} \mathrm{~g}^{-1} \mathrm{LDW}\right)$ and root: shoot ratio.

Table 6: Effect of water stress treatments on leaf area ratio $\left(\mathrm{cm}^{2} \mathrm{~g}^{-1} \mathrm{TDW}\right)$, specific leaf area $\left(\mathrm{cm}^{2} \mathrm{~g}^{-1}\right.$ LDW) and root:shoot ratio.

\begin{tabular}{lcccccccccccc}
\hline Accession & \multicolumn{3}{c}{ Leaf area ratio $\left(\mathrm{cm}^{2} \mathrm{~g}^{-1} \mathrm{TDW}^{*}\right)$} & \multicolumn{3}{c}{ Specific leaf area $\left(\mathrm{cm}^{2} \mathrm{~g}^{-1} \mathrm{LDW}^{* *}\right)$} & \multicolumn{4}{c}{ Root: shoot ratio } \\
\cline { 2 - 15 } & \multicolumn{3}{c}{ Irrigation treatments } & \multicolumn{4}{c}{ Irrigation treatments } & \multicolumn{4}{c}{ Irrigation treatments } \\
& $100 \%$ & $75 \%$ & $100 \%$ & $25 \%$ & $100 \%$ & $75 \%$ & $50 \%$ & $25 \%$ & $100 \%$ & $75 \%$ & $50 \%$ & $25 \%$ \\
\hline GKU-37 & 80.30 & 86.30 & 74.83 & 82.73 & 284.16 & 317.30 & 272.91 & 451.28 & 0.72 & 0.64 & 0.60 & 0.65 \\
GKU-24 & 84.39 & 90.69 & 79.22 & 87.12 & 307.30 & 340.44 & 384.83 & 563.20 & 0.85 & 0.77 & 0.73 & 0.78 \\
LUT-45 & 75.93 & 82.23 & 70.76 & 78.66 & 282.91 & 316.05 & 360.44 & 539.11 & 0.70 & 0.62 & 0.58 & 0.63 \\
BNU-10 & 82.93 & 89.23 & 77.76 & 85.66 & 251.28 & 284.42 & 328.81 & 507.18 & 0.79 & 0.71 & 0.67 & 0.72 \\
ASM-124 & 72.11 & 78.41 & 66.94 & 74.84 & 267.83 & 300.97 & 345.36 & 523.73 & 0.68 & 0.60 & 0.56 & 0.61 \\
Mean & 79.13 & 85.38 & 73.90 & 81.80 & 278.70 & 311.84 & 338.47 & 516.90 & 0.75 & 0.67 & 0.63 & 0.68 \\
SD & 4.54 & 2.24 & 4.52 & 4.52 & 18.63 & 18.63 & 37.60 & 37.64 & 0.06 & 0.06 & 0.06 & 0.06 \\
CV & 0.06 & 0.03 & 0.06 & 0.05 & 0.07 & 0.06 & 0.11 & 0.07 & 0.08 & 0.09 & 0.09 & 0.09 \\
SEM & 2.03 & 1.00 & 2.01 & 2.01 & 16.80 & 8.32 & 16.78 & 16.80 & 0.02 & 0.02 & 0.02 & 0.02 \\
& \multicolumn{3}{c}{ LSD value at $0.05=0.09$} & \multicolumn{3}{c}{ LSD value at $0.05=31.36$} & LSD value at $0.05=0.0$ (NS) \\
\hline
\end{tabular}

TDW-Total Dry Matter Weight, **LDW-Leaf Dry Matter Weight

$\mathrm{SD}=$ Standard deviation; $\mathrm{CV}=$ coefficient of variance; $\mathrm{SEM}=\mathrm{Standard}$ error of mean 
Table 7: Effect of water stress treatments on biomass partitioning to shoot (\%), biomass partitioning to root $(\%)$ and biomass partitioning to leaves $(\%)$.

\begin{tabular}{|c|c|c|c|c|c|c|c|c|c|c|c|c|}
\hline \multirow{3}{*}{$\begin{array}{l}\text { Accession } \\
\text { Code }\end{array}$} & \multicolumn{4}{|c|}{ Biomass partitioning to shoot (\%) } & \multicolumn{4}{|c|}{ Biomass partitioning to root (\%) } & \multicolumn{4}{|c|}{ Biomass partitioning to leaves (\%) } \\
\hline & \multicolumn{4}{|c|}{ Irrigation treatments } & \multicolumn{4}{|c|}{ Irrigation treatments } & \multicolumn{4}{|c|}{ Irrigation treatments } \\
\hline & $100 \%$ & $75 \%$ & $50 \%$ & $25 \%$ & $100 \%$ & $75 \%$ & $50 \%$ & $25 \%$ & $100 \%$ & $75 \%$ & $50 \%$ & $25 \%$ \\
\hline GKU-37 & 52.9 & 49.9 & 46.6 & 43.2 & 33.2 & 29.6 & 28.7 & 28.6 & 26.4 & 24.0 & 20.6 & 13.1 \\
\hline GKU-24 & 58.9 & 55.9 & 52.6 & 49.2 & 33.0 & 36.0 & 35.1 & 35.0 & 29.7 & 27.3 & 23.9 & 16.4 \\
\hline LUT-45 & 50.4 & 47.4 & 44.1 & 40.7 & 26.2 & 22.6 & 21.7 & 21.6 & 28.2 & 25.8 & 22.4 & 14.9 \\
\hline BNU-10 & 56.0 & 53.0 & 49.7 & 46.3 & 33.0 & 29.4 & 28.5 & 28.4 & 24.3 & 21.9 & 18.5 & 11.0 \\
\hline ASM-124 & 57.8 & 54.8 & 51.5 & 48.1 & 34.0 & 30.4 & 29.8 & 29.4 & 15.9 & 13.5 & 10.1 & 4.6 \\
\hline Mean & 55.2 & 52.2 & 48.9 & 45.5 & 31.9 & 29.6 & 28.8 & 28.6 & 25.0 & 22.5 & 19.1 & 12.0 \\
\hline SD & 3.14 & 3.14 & 3.14 & 3.14 & 2.86 & 4.26 & 4.27 & 4.26 & 4.82 & 4.84 & 4.85 & 4.12 \\
\hline $\mathrm{CV}$ & 0.05 & 0.06 & 0.06 & 0.06 & 0.09 & 0.14 & 0.14 & 0.15 & 0.19 & 0.21 & 0.25 & 0.34 \\
\hline \multirow[t]{2}{*}{ SEM } & 1.40 & 1.40 & 1.40 & 1.40 & 1.77 & 1.90 & 1.91 & 1.90 & 2.15 & 2.16 & 2.16 & 1.84 \\
\hline & \multicolumn{4}{|c|}{ LSD value at $0.05=0.0(\mathrm{NS})$} & \multicolumn{4}{|c|}{ LSD value at $0.05=2.01$} & \multicolumn{4}{|c|}{ LSD value at $0.05=0.61$} \\
\hline
\end{tabular}

$\mathrm{SD}=$ Standard deviation; $\mathrm{CV}=$ coefficient of variance $; \mathrm{SEM}=$ Standard error of mean

Table 8: Effect of water stress treatments on leaves nitrogen percentage, leaves phosphorus percentage and leaves potassium percentage.

\begin{tabular}{lcccccccccccc}
\hline \multirow{2}{*}{$\begin{array}{l}\text { Accession } \\
\text { Code }\end{array}$} & \multicolumn{3}{c}{ Leaf N\% } & \multicolumn{4}{c}{ Irrigation treatments } & \multicolumn{4}{c}{ Irrigation treatments } & \multicolumn{4}{c}{ Leaf K\% } \\
\cline { 2 - 13 } & $100 \%$ & $75 \%$ & $50 \%$ & $25 \%$ & $100 \%$ & $75 \%$ & $50 \%$ & $25 \%$ & $100 \%$ & $75 \%$ & $50 \%$ & $25 \%$ \\
\hline GKU-37 & 2.30 & 2.15 & 2.10 & 2.05 & 0.37 & 0.30 & 0.30 & 0.26 & 1.10 & 1.00 & 0.93 & 0.92 \\
GKU-24 & 2.16 & 2.13 & 2.13 & 2.20 & 0.42 & 0.32 & 0.30 & 0.30 & 1.12 & 1.05 & 0.93 & 0.90 \\
LUT-45 & 2.13 & 2.10 & 2.00 & 1.90 & 0.27 & 0.27 & 0.20 & 0.20 & 0.93 & 0.82 & 0.75 & 0.73 \\
BNU-10 & 2.04 & 2.02 & 2.00 & 1.95 & 0.35 & 0.35 & 0.34 & 0.25 & 0.98 & 0.90 & 0.75 & 0.73 \\
ASM-124 & 2.07 & 1.80 & 1.78 & 1.78 & 0.40 & 0.33 & 0.25 & 0.15 & 0.77 & 0.70 & 0.73 & 0.70 \\
Mean & 2.14 & 2.04 & 2.00 & 1.97 & 0.36 & 0.31 & 0.28 & 0.23 & 0.98 & 0.91 & 0.82 & 0.80 \\
SD & 0.09 & 0.13 & 0.12 & 0.14 & 0.05 & 0.03 & 0.05 & 0.05 & 0.13 & 0.13 & 0.09 & 0.09 \\
CV & 0.04 & 0.06 & 0.06 & 0.07 & 0.14 & 0.10 & 0.18 & 0.22 & 0.13 & 0.14 & 0.11 & 0.11 \\
SEM & 0.04 & 0.06 & 0.05 & 0.06 & 0.02 & 0.01 & 0.02 & 0.02 & 0.06 & 0.06 & 0.04 & 0.04 \\
& \multicolumn{3}{c}{ LSD value at 0.05=0.09 } & \multicolumn{3}{c}{ LSD value at $0.05=0.05$} & & LSD value at $0.05=0.05$ \\
\hline
\end{tabular}

$\mathrm{SD}=$ Standard deviation; $\mathrm{CV}=$ coefficient of variance; $\mathrm{SEM}=$ Standard error of mean

GKU-24 seemed to have more P content compared to the other accessions at irrigation with $25 \%$ from field capacity. The obtained results were in agreement with those obtained by Moustafa (2002) who concluded that the leaf $\mathrm{P}$ content increased as amount of applied water was increased in olive trees. A good supply of water is required for phosphate availability and absorption by plants. Phosphate ions move through soils primarily through diffusion and if the water content in the soil decreases, the radii of waterfilled pores decrease, tortuosity increases and P mobility decreases (Faye et al., 2006). Drought causes a reduction in $\mathrm{P}$ absorption and transport in plants. A decrease in available $\mathrm{P}$ forms and increase in occluded $\mathrm{P}$ in the soil reduces $\mathrm{P}$ uptake and consequently induces lower foliar P content (Sardans and Peñuela, 2004). Data presented in Table 8 also indicated that potassium content in leaves was gradually decreased by decreasing water depletion from $100 \%$ to $25 \%$ from field capacity. In this regard, ASM-124 produced lower K $(0.70 \%)$ content compared to the other accessions when irrigated at $25 \%$ from field capacity. These findings are generally in line with previous reports that the leaves of olive contained a lower content of K when these plants are grown under severe water stress (Gowda, 1998; Hassan, 1998). Water conditions in plants influence the $\mathrm{K}^{+}$accumulation in leaves and interact with $\mathrm{K}^{+}$nutritional status in some plant species (Restrepo-Diaz et al., 2008). The stomatal opening mechanism is governed by the $\mathrm{K}^{+}$ concentration (Mengel and Kirkby, 2001; Larcher, 2006; Taiz and Zeiger, 2006; Mengel, 2007). The 
opening and closure of $\mathrm{K}^{+}$channels are of particular importance to guard cells and this action mechanism is controlled by the reception of red light, which induces stomatal opening (Mengel, 2007). Under mild water stress, plants tend to reduce the stomata aperture and when water stress becomes severe, the stomata generally close (Larcher, 2006). The effect of irrigation treatments on leaf Ca content was significant as shown in Table 9. Results indicated that leaf Ca content decreased by increasing drought stress. GKU-24 recorded the highest significant value $(0.20 \%)$ of leaf Ca content, while ASM-124 recorded the lowest significant value $(0.11 \%)$ when irrigated at $25 \%$ from field capacity. These findings are in agreement with those obtained by Moustafa (2002) and Nomir (1994) where they concluded that leaf Ca content was increased as amount of applied water was increased. Analyzing the long-term effects of drought in a Mediterranean evergreen (Quercus ilex) forest, Sardans et al. (2008) concluded that drought tended to decrease $\mathrm{Ca}$ concentrations in the aboveground biomass and this effect was attributed to the reduction in transpiration flux. The Na content in leaves gradually decreased by decreasing water depletion from $100 \%$ to $25 \%$ from field capacity (Table 9 ).

Table 9: Effect of water stress treatments on leaves sodium percentage, leaves chloride percentage and leaves calcium percentage.

\begin{tabular}{lcccccccccccc}
\hline \multirow{2}{*}{$\begin{array}{l}\text { Accession } \\
\text { Code }\end{array}$} & \multicolumn{3}{c}{ Leaf Na percentage } & \multicolumn{4}{c}{ Leaf Cl percentage } & \multicolumn{3}{c}{ Leaf Ca percentage } \\
\cline { 2 - 14 } & $100 \%$ & $75 \%$ & $50 \%$ & $25 \%$ & $100 \%$ & $75 \%$ & $50 \%$ & $25 \%$ & $100 \%$ & $75 \%$ & $50 \%$ & $25 \%$ \\
\hline GKU-37 & 0.22 & 0.22 & 0.20 & 0.14 & 0.35 & 0.22 & 0.25 & 0.20 & 0.30 & 0.25 & 0.23 & 0.20 \\
GKU-24 & 0.30 & 0.20 & 0.20 & 0.20 & 0.52 & 0.31 & 0.25 & 0.25 & 0.30 & 0.26 & 0.25 & 0.20 \\
LUT-45 & 0.23 & 0.15 & 0.14 & 0.13 & 0.23 & 0.17 & 0.13 & 0.13 & 0.24 & 0.20 & 0.18 & 0.12 \\
BNU-10 & 0.25 & 0.17 & 0.17 & 0.13 & 0.30 & 0.23 & 0.22 & 0.20 & 0.25 & 0.23 & 0.20 & 0.17 \\
ASM-124 & 0.20 & 0.12 & 0.10 & 0.05 & 0.34 & 0.30 & 0.25 & 0.25 & 0.23 & 0.20 & 0.16 & 0.11 \\
Mean & 0.24 & 0.17 & 0.16 & 0.13 & 0.34 & 0.24 & 0.22 & 0.20 & 0.26 & 0.23 & 0.20 & 0.16 \\
SD & 0.09 & 0.03 & 0.04 & 0.05 & 0.15 & 0.05 & 0.05 & 0.04 & 0.03 & 0.02 & 0.10 & 0.03 \\
CV & 0.56 & 0.17 & 0.25 & 0.38 & 0.57 & 0.21 & 0.23 & 0.20 & 0.11 & 0.08 & 0.50 & 0.19 \\
SEM & 0.04 & 0.01 & 0.07 & 0.02 & 0.06 & 0.02 & 0.02 & 0.02 & 0.01 & 0.01 & 0.04 & 0.01 \\
& \multicolumn{3}{c}{ LSD value at $0.05=0.05$} & \multicolumn{3}{c}{ LSD value at $0.05=0.09$} & & LSD value at $0.05=0.01$
\end{tabular}

$\mathrm{SD}=$ Standard deviation; $\mathrm{CV}=$ coefficient of variance; $\mathrm{SEM}=$ Standard error of mean

However, the lowest $\mathrm{Na}$ content $(0.05 \%)$ was produced when ASM-124 was exposed to severe drought stress $(25 \%)$, while the highest $(0.22 \%)$ when GKU-37 was exposed to moderate drought stress (75\%) in comparison to the other accessions under the same drought conditions. These results go in parallel with those obtained by Nomir (1994) who illustrated that the decrease in soil moisture level decreased $\mathrm{Na}$ content in persimmon leaves. The $\mathrm{Cl}$ content in leaves gradually decreased by decreasing water depletion from 100 to $25 \%$ from field capacity (Table 9). However, the highest $\mathrm{Cl}$ content $(0.31 \%)$ was found when GKU-24 was exposed to moderate drought stress $(75 \%)$, while the lowest $(0.13 \%)$ when LUT-45 was exposed to $25 \%$ drought in comparison to the other accessions under the same drought conditions. These results were found to be in harmony with Nomir (1994) who illustrated that the reduction in soil moisture level caused decrease in concentrations of $\mathrm{Cl}$ content in persimmon leaves. Chloride ion is involved in the photolysis of water by photosystem. It is required for cell division in both leaves and roots, plays an important role in stomatal movement and has important functions in osmoregulation and the water relation.

Data presented in Table 10 indicated that proline accumulation in teak plants increased gradually with increasing the level of drought stress up to $25 \%$ in case of GKU-24 and GKU-37. The highest proline accumulation was obtained with GKU-24 when subject under severe drought stress condition (25\%) in comparison to normal irrigation and also to other drought stresses (50\% and $75 \%)$. Under 
drought conditions, the accumulations of proline seemed to be associated with drought tolerance in many plant species (Liu et al., 2011). Proline accumulation may play a role in minimising the damage caused by dehydration (Mohammadkhani and Heidari, 2008). Proline is an amino acid and known to act as an osmolyte, protecting the plasma membrane integrity (Mansour, 1998), as a sink of energy or reducing power (Verbruggen et al., 1996), as a scavenger of ROS and their derivatives (Hong et al., 2000; Bashir et al., 2007) and as a source for carbon and nitrogen (Peng et al., 1996), and thereby helping the plants to tolerate stress effects (Manivannan et al., 2007). The results in Table 10 also showed that chlorophyll content was affected by drought conditions. GKU-24 recorded the highest significant value (0.70), while LUT-45 and ASM-124 recorded the lowest values (0.57 and 0.58 respectively) under severe drought stress condition (25\%). Similar results are in agreement with those obtained by Liu et al. 2011; Ghaderi and Siosemardeh 2011; Dias and Bruggemann 2010; who found that chlorophyll was decreased with increasing water stress. The decrease in chlorophyll content has been considered to be a typical symptom of oxidative stress and may be the result of pigment photo-oxidation, chlorophyll degradation and/or chlorophyll synthesis deficiency (Smirnoff, 1993). Munne-Bosch and Alegre 2000; Galmes et al. 2007; Elsheery and Cao 2008 explained this phenomenon as a photoprotection mechanism through reducing light absorbance by decreasing pigments content.

Table 10: Effect of drought treatments on leaf proline ( $\mathrm{mg} / \mathrm{g}$ fresh weight) content and total chlorophyll content.

\begin{tabular}{|c|c|c|c|c|c|c|c|c|}
\hline \multirow{3}{*}{$\begin{array}{l}\text { Accession } \\
\text { Code }\end{array}$} & \multicolumn{4}{|c|}{ Leaf proline } & \multicolumn{4}{|c|}{ Total chlorophyll content } \\
\hline & \multicolumn{4}{|c|}{ Irrigation treatment } & \multicolumn{4}{|c|}{ Irrigation treatments } \\
\hline & $100 \%$ & $75 \%$ & $50 \%$ & $25 \%$ & $100 \%$ & $75 \%$ & $50 \%$ & $25 \%$ \\
\hline GKU-37 & 0.015 & 0.016 & 0.020 & 0.026 & 0.67 & 0.67 & 0.65 & 0.64 \\
\hline GKU-24 & 0.016 & 0.023 & 0.024 & 0.033 & 0.75 & 0.71 & 0.71 & 0.70 \\
\hline LUT-45 & 0.014 & 0.016 & 0.017 & 0.018 & 0.70 & 0.64 & 0.60 & 0.58 \\
\hline BNU-10 & 0.012 & 0.014 & 0.017 & 0.021 & 0.68 & 0.66 & 0.65 & 0.57 \\
\hline ASM-124 & 0.012 & 0.015 & 0.018 & 0.023 & 0.66 & 0.66 & 0.61 & 0.60 \\
\hline Mean & 0.013 & 0.016 & 0.019 & 0.024 & 0.69 & 0.67 & 0.64 & 0.62 \\
\hline SD & 0.003 & 0.003 & 0.002 & 0.007 & 0.03 & 0.02 & 0.04 & 0.05 \\
\hline $\mathrm{CV}$ & 0.143 & 0.143 & 0.105 & 0.318 & 0.04 & 0.03 & 0.06 & 0.08 \\
\hline \multirow[t]{2}{*}{ SEM } & 0.001 & 0.001 & 0.001 & 0.004 & 0.01 & 0.01 & 0.02 & 0.02 \\
\hline & \multicolumn{4}{|c|}{ LSD value at $0.05=0.01$} & \multicolumn{4}{|c|}{ LSD value at $0.05=0.03$} \\
\hline
\end{tabular}

$\mathrm{SD}=$ Standard deviation; $\mathrm{CV}=$ coefficient of variance; $\mathrm{SEM}=$ Standard error of mean

\section{Conclusion}

Limited availability of water is the major contributor to the forest decline and thus has significant effect on global forest cover (Bigler et al., 2006; van Mantgem et al., 2009; Allen et al., 2010). However, like other crop plant species, tree species also developed strategies to cope with limited water supply. Understanding these strategies including physiological and biochemical responses is vital to meet the challenges imposed by the global climate change phenomena including drought. Decreasing the irrigation water, decreased the plant height, leaves number/accessions, leaf area, leaf content of $\mathrm{N}, \mathrm{P}, \mathrm{K}, \mathrm{Ca}, \mathrm{Na}$ and $\mathrm{Cl}$. Accumulation of proline was also significantly affected by drought stress condition, which increased with increasing water deficit. Similarly, the transpiration rate, stomatal conductance, net photosynthetic rate, relative water content, intrinsic and instantaneous water use efficiency, plant biomass and chlorophyll content were also significantly affected by drought stress condition. Water deficit did not significantly affect the leaf area ratio however, the specific leaf area increased significantly in plants submitted to decreasing irrigation water. Root: shoot ratio also did not differ significantly due to water deficit. GKU-24, GKU-37 and BNU-10 were found to be more tolerant to drought stress compared to the 
other studied accessions. ASM-124 and LUT-45 showed more sensitivity towards drought and affected mostly by drought and the vegetative, physiological and chemical characteristics of accessions were found to be lower as compared to the other accessions. GKU-24 was found to be the highest tolerance against drought followed by GKU-37 and BNU-10. These sources can safely be used for large-scale reforestation programmes in the region. Germplasm used in afforestation programmes in India and other countries, generally utilizes only locally available material. Thus opportunities for using materials with high drought tolerance potential or with more desirable characteristics might have been missed. This work will facilitate selection of promising sources for multi-location evaluation and will also hasten the process of utilisation of germplasm. It further gives a direction to the effect and practice studies for genetic improvement of this species.

\section{Acknowledgment}

The authors are grateful to the Director, CSIR-North East Institute of Science and Technology, Jorhat, Assam, India for his permission to publish the work. SPS is thankful to Department of Biotechnology (DBT), Government of India for financial support. S.P.S. thanks the anonymous reviewers for their insightful suggestions and critical reviews.

\section{References}

Abd El-Samed, G.A., 1995. Effect of irrigation regimes on growth, yield and water use of olive trees. Ph.D. Thesis, Fac. Agric., Fayoum, Cairo Univ., Egypt.

Allen, C.D., Macalady, A.K., Chenchouni, H., Bachelet, D., McDowell, N., Vennetier, M., Kitzberger, T., Rigling, A., Breshears, D.D. and Hogg, E.T., 2010. A global overview of drought and heatinduced tree mortality reveals emerging climate change risks for forests. For Ecol Manag, 259(4): 660-684.

Bänziger, M., Edmeades, G.O., Beck, D. and Bellon, M., 2000. Breeding for Drought and Nitrogen Stress Tolerance in Maize: From Theory to Practice. CIMMYT, Mexico, D.F., 68.

Bashir, F., Mahmooduzzafar Siddiqi T.O. Iqb and al, M., 2007. The antioxidative response system in soybean plants exposed to Deltamethrin, a synthetic pyrethroid insecticide. Environmental Pollution, 147:94-100.

Bates, L.S., Waldren, R.P. and Teare, I.D., 1973. Rapid determination of free proline for water-stress studies. Plant and Soil, 939:205-207.

Bigler, C., Bräker, O.U., Bugmann, H., Dobbertin, M. and Rigling, A., 2006. Drought as an inciting mortality factor in scots pine stands of the Valais, Switzerland. Ecosystems, 9:330-343.

Bolhar-Nordenkampf, H.R., 1987. Shoot morphology and leaf anatomy in relation to photosynthesis. In: Coombs J, Hall DO, Long SP, Scurlock JMO (ed) Techniques in Bioproductivity and photosynthesis, Pergamon Press, Oxford.

Brown, J.D. and Lilleland, O., 1946. Rapid determination of potassium and sodium in plant material and soil extract by Flame Photometry. Proc Amer Soc Hort Sci, 73:813.

DaMatta, F., Loos, R.A., Silva, E.A., Loureiro, M.E. and Ducatti, C., 2002. Effects of soil water déficit and nitrogen nutrition on water relations and photosynthesis of pot-grown Coffea canephora Pierra. Trees, 16:555-558.

Dias, M.C. and Bruggemann, W., 2010. Limitations of photosynthesis in Phaseolus vulgaris under drought stress: gas exchange, chlorophyll fluorescence and Calvin cycle enzymes. Photosynthetica, 48:96-102.

Duan, B.L., Lu, Y.W., Yin, C.Y., Junttila, O. and Li, C.Y., 2005. Physiological responses to drought and shade in two contrasting Picea asperata populations. Physiol Plant, 124:476-484.

Elsheery, N.I. and Cao, K.F., 2008. Gas exchange, chlorophyll fluorescence and osmotic adjustment in two mango cultivars under drought stress. Acta Physiol Plant, 30:769-777. 
Farooq, M., Basra, S.M.A., Wahid, A., Cheema, Z.A., Cheema, M.A. and Khaliq, A., 2008. Physiological role of exogenously applied glycinebetaine in improving drought tolerance of fine grain aromatic rice (Oryza sativa L.). Journal of Agronomy and Crop Science, 194:325-333.

Faye, I., Diouf, O., Guissé, A., Sène, M. and Diallo, N., 2006. Characterising root responses to low phosphorus in pearl millet [Pennisetum glaucum (L.) R. Br.]. Agronomy Journal, 98:1187-1194.

Fordyce, I.R., Duff, G.A. and Eamus, D., 1995. The ecophysiology of Allosyncarpia ternate (Myrtaceae) in Northern Australia: Tree physiognomy, leaf characteristics and assimilation at contrasting sites. Australian Journal of Botany, 43:367-377.

Galmes, J., Abadia, A., Medrano, H. and Flexas, J., 2007. Photosynthesis and photoprotection responses to water stress in the wild-extinct plant Lysimachia minoricensis. Environmental and Experimental Botany, 60:308-317.

Ghaderi, N. and Siosemardeh, A., 2011. Response to drought stress of two strawberry cultivars (cv. Kurdistan and Selva). Horticulture, Environment, and Biotechnology, 52:6-12.

Gowda, M.A., 1998. Studies of sensitivity of some olive varieties to drought. Dissertation, M. Sc., Hort Dept, Fac Agric, Minia University, Egypt.

Greenwood, D.R., Scarr, M.J. and Christophel, D.C., 2003. Leaf stomatal frequency in the Australian tropical tree Neolitsea dealbata (Lauraceae) as a proxy measure of atmospheric $\mathrm{CO}_{2}$. Palaeoecology, 196:375-393.

Hassan, A.A., 1998. Effect of drought on fruit seedlings. Dissertation, M. Sc., Al-Azahar University, Cairo, Egypt.

Hiscox, J.D. and Isrealstam G.F., 1979. A method for extraction of chlorophyll from leaf tissues without maceration. Canadian Journal of Botany, 57:1332-1334.

Hong, Z., Lakkineni, K. and Zhang K., Verma D.S.P., 2000. Removal of feedback inhibition of ^1pyrroline-5-carboxylate synthetase results in increased proline accumulation and protection of plants from osmotic stress. Plant Physiology, 122:1129-1136.

Hunt, R., 1990. Basic Growth Analysis. Unwin Hyman Ltd., London.

Jackson, M.L., 1958. Soil Chemical Analysis. Prentice-Hall, Inc. Englewood Cliffs, USA.

Jaleel, C.A., Gopi, R., Sankar, B., Gomathinayagam, M. and Panneerselvam, R., 2008a. Differential responses in water use efficiency in two varieties of Catharanthus roseus under drought stress.

Comptes Rendus Biologies, 331:42-47.

Jaleel, C.A., Sankar, B., Murali, P.V., Gomathinayagam, M., Lakshmanan, G.M.A. and Panneerselvam, R., 2008b. Water deficit stress effects on reactive oxygen metabolism in Catharanthus roseus, impacts on ajmalicine accumulation. Colloids Surf B: Biointerface, 62:105-111.

Klute, A., 1986. Methods for Soil Analysis, Part 1, Physical and Mineralogical Methods, Second Edition. American Society of Agronomy, Agronomy Monographs 9, Madison, Wisconsin, 1188.

Kusaka, M., Ohta, M. and Fujimura, T., 2005. Contribution of inorganic components to osmotic adjustment and leaf folding for drought tolerance in pearl millet. Physiologia Plantarum, 125:474489.

Larcher, W. (2006). Physiological Plant Ecology. $4^{\text {th }}$ ed. Rima, São Carlos, 50.

Liu, C., Liu, Y., Guo, K., Fan, D., Li, G., Zheng, Y., Yu, L. and Yang, R., 2011. Effect of drought on pigments, osmotic adjustment and antioxidant enzymes in six woody plant species in karst habitats of south western China. Environmental and Experimental Botany, 71:174-183.

Manivannan, P., Abdul, Jaleel C., Sankar, B., Kishorekumar, A., Somasundaram, R., Lakshmanan ,G.M.A., Panneerselvam, R., 2007. Growth, biochemical modifications and proline metabolism in Helianthus annuus L. as induced by drought stress. Colloids Surfaces B: Biointerfaces, 59:141149. 
Mansour, M.M.F., 1998. Protection of plasma membrane of onion epidermal cells by glycine betaine and proline against $\mathrm{NaCl}$ stress. Plant Physiology and Biochemistry, 36:767-772.

Mengel, K., 2007. Potassium. In Handbook of Plant Nutrition. $1^{\text {st }}$ ed. CRC Taylor and Francis, NY, 91120.

Mengel, K. and Kirkby, E.A., 2001. Principles of Plant Nutrition, $4^{\text {th }}$ ed. International Potash Institute, Switzerland, 687.

Mohammadkhani, N. and Heidari, R., 2008. Drought-induced accumulation of soluble sugars and proline in two maize varieties. World Applied Sciences Journal, 3:448-453.

Moustafa, A.H.A., 2002. Studies on the irrigation of olive trees grown in new reclaimed soils. Ph.D. Thesis Fac. Agric., Zagazig University, Egypt.

Munne-Bosch, S. and Alegre, L., 2000. Changes in carotenoids, tocopherols and diterpenes during drought and recovery and the biological significance of chlorophyll loss in Rosmarinus officinalis plants. Planta, 207:925-931.

Nomir S.A., 1994. Physiological studies on kaki. Ph. D. Thesis., Fac. Agric. Zagazig University, Egypt.

Novozamsky, I., van, Eck R., Ch. van Schouwenburg, J. and Walinga, I., 1974. Total nitrogen determination in plant material by means of the indophenol blue method. Netherlands Journal of Agricultural Science, 22:3-5.

Panse, V. G. and Shukhatme, P.V., 1967. Statistical procudures for Agricultural Worker, ICAR, New Delhi.

Peng Z. and Lu Q., Verma D.P., 1996. Reciprocal regulation of ^1-pyrroline-5-carboxylate synthetase and proline dehydrogenase genes controls proline levels during and after osmosis stress in plants. Molecular Genetics and Genomics, 253:334-341.

Petite, M.A., Moro, G.B., Murua, G.C., Lacuesta, M. and Rueda, M.A., 2000. Sequential effects of acidic precipitation and drought on photosynthesis and chlorophyll fluorescence parameters of Pinus radiate D. Don seedlings. Journal of Plant Physiology, 156:84-92.

Piper, C.S., 1950. Soil and Plant Analysis. International Science Publisher, Inc. New York. 368.

Radford, P.J., 1967. Growth analysis formulae - their use and abuse. Crop Science, 7:171-175.

Restrepo-Diaz, H., Benlloch, M. and Fernández-Escobar, R., 2008. Plant water stress and K + starvation reduce absorption of foliar applied K + by olive leaves. Scientia Horticulturae, 116:409-413.

Sardans, J. and Penuelas, J., 2004. Increasing drought decreases phosphorus availability in an evergreen Mediterranean forest. Plant Soil, 267:367-377.

Sardans, J., Peñuelas, J. and Ogaya, R., 2008. Drought's impact on Ca, Fe, Mg, Mo and S concentration and accumulation patterns in the plants and soil of a Mediterranean evergreen Quercus ilex forest. Biogeochemistry, 87:49-69.

Shao, H.B., Chu, L.Y., Shao, M.A., Abdul, Jaleel C. and Hong-Mei, M., 2008. Higher plant antioxidants and redox signaling under environmental stresses. Comp Rend Biol, 331:433-441.

Shaheen, M.A., Hegaz, A.A. and Hmmam, I.S.A., 2011. Effect of water stress on vegetative characteristics and leaves chemical constituents of some transplants Olive cultivars. AmericanEurasian Journal of Agriculture and Environmental Science, 11:663-670.

Smirnoff, N., 1993. The role of active oxygen in the response to water deficit and desiccation. New Phytologist, 125:27-58.

Taiz, L.and Zeiger, E., 2002. Plant Physiology, $3^{\text {rd }}$ edn. Sinauer Associates, Inc., Publishers, Massachusetts.

Taiz, L. and Zeiger, E., 2006. Plant Physiology. $4^{\text {th }}$ ed. Sinauer Associates, Inc., Publishers, Massachusetts.

Temminhhoff, E.E.J.M. and Houba, V.J.G., 2004. Plant Analysis Procedures. Second Edition, Kluwer Academic Publishers. Dordrecht, Boston, London, 179. 
Borah et al. /Journal of Tropical Forestry and Environment Vol. 9, No. 02 (2019) 21-35

Vaishnaw, V., Mohammad, N., Wali, S.A., Kumar, R., Tripathi, S.B., Negi, M.S. and Ansari, S.A., 2015. AFLP markers for analysis of genetic diversity and structure of teak (Tectona grandis) in India. Canadian Journal of Forest Research, 45:297-306.

van Mantgem, P.J., Stephenson, N.L., Byrne, J.C., Daniels, L.D., Franklin, J.F., Fule, P.Z., Harmon, M.E., Larson, A.J., Smith, J.M., Taylor, A.H. and Veblen, T.T., 2009. Widespread increase of tree mortality rates in the western United States. Science, 323:521-524.

Verbruggen, N., Hua, X.J., May, M.M. and Vanmontagu, M., 1996. Environmental and developmental signals modulate proline homoeostasis: Evidence for a negative transcriptional regulator. Proceedings of the National Academy of Sciences of the United States of America, USA 93, 87878791.

Verhaegen, D., Fofana, I.J., Logossa, Z.A. and Ofori, D., 2010. What is the genetic origin of teak (Tectona grandis L.) introduced in Africa and in Indonesia? Tree Genetics and Genomes, 6:717733.

Weatherley, P.E., 1950. Studies in the water relations of the cotton plant. I. The field measurement of water deficits in leaves. New Phytologist, 49:81-87. 